\title{
RATGEBERLITERATUR UND STRATEGIEN DER NORMIERUNG - AM BEISPIEL VON WISSENSCHAFTSRATGEBERN
}

\begin{abstract}
Advice Literature and Strategies of Normativization
This article is going to follow up the question of normativization within counsellor texts, exemplarily using science guides. A pro argumentation for this is, to not only see normativization in this correlation as a morpho-syntactically and lexical medium - as we usually would - but beyond that also include patterned text structures. These are to be interpreted as appellative (and persuasive) strategies of the authors instance which ought to establish a relationship to the readers who are supposed to be guided by this foundation.
\end{abstract}

KEYWORDS: Advice Literature - Normativization

\section{Einleitung}

Im vorliegenden Beitrag wird der Frage nach der Normativität bzw. Normierung in Ratgeberliteratur nachgegangen. Genau genommen werden mit einem diachronen Blick auf Ratgeber zum wissenschaftlichen Arbeiten textstrukturelle Muster herausgearbeitet, die sich als historisch spezifische Normierungsstrategien interpretieren lassen.

Aufbauend auf den Überlegungen von Christa Pieth und Kirsten Adamzik (1997) sowie meinen eigenen Untersuchungen (Niemann i. V.) geht es hier also grundsätzlich darum, die Normierung in Wissenschaftsratgebern als ein vielschichtiges, auf mehreren Ebenen produktives Phänomen zu betrachten: Neben der in diesem Zusammengang eher üblichen Betrachtung von einerseits morpho-syntaktischen Mitteln wie Imperativen und Modalverben oder andererseits von bestimmten lexikalischen Mitteln scheint Normierung auch mit Blick auf komplexere Ebenen wie diejenigen der textuellen Strukturierung erfassbar zu sein. Zumindest andeutungsweise soll dies im vorliegen-

Robert Niemann - Justus-Liebig-Universität, Gießen, robert.niemann@germanistik.uni-giessen.de 
den Beitrag gezeigt werden, wobei die Ausführungen insgesamt noch stark vorläufigen Charakter haben ${ }^{1}$.

Es wird dabei wie folgt vorgegangen: Zunächst einmal werden die folgenden Ausführungen in Zusammenhang zur einschlägigen Forschung zu (Sprach-)Ratgeberliteratur gebracht. Es wird dabei im Kern darum gehen, mit den Fragen nach den Untersuchungszeiträumen sowie der Normativität zwei zentrale Forschungsdesiderata in dieser vergleichsweise jungen linguistischen Forschungsrichtung aufzuzeigen (vgl. dazu etwa Greule, 2000; Kessel, 2009). Im Anschluss daran werden zunächst die spezifischen Voraussetzungen zum Thema Wissenschaftsratgeber geklärt, woraufhin schließlich eine kleine diachrone Analyse von Beispielen folgt.

\section{Ausgangspunkte - (Sprach-)Ratgeber}

Die sprachwissenschaftliche Beschäftigung mit dem Thema Sprachratgeber ${ }^{2}$ kann als vergleichsweise jung betrachtet werden. Albrecht Greule etwa stellt noch Anfang der 2000er Jahre heraus, dass der Gegenstand erst allmählich auf linguistisches Interesse zu stoßen scheint: „Aus sprachwissenschaftlicher Perspektive muss man feststellen, daß die Sprachratgeber - mit geringfügigen Ausnahmen - für germanistische Linguisten kein Thema waren, es aber, wenn mich der Schein nicht trügt, zu werden beginnen“ (Greule, 2000, S. 317). Bei dieser jungen Beschäftigung mit Ratgeberliteratur lassen sich offenbar zwei Akzente schwerpunktmäßig herausstellen: die Frage nach der Zeit sowie die Frage nach der Normativität ${ }^{3}$.

Zunächst einmal scheint das Interesse an Sprachratgebern zum Großteil sprachhistorisch motiviert zu sein. Katja Kessel sieht die Gründe hierfür in erster Linie in dem Potential von Sprachratgebern, Auskünfte über das typische Sprach- und vor allem Sprechverhalten in früheren Epochen geben zu können (vgl. Kessel, 2009, S. 23). Sprachratgeber dienen insofern als Quellen für historische Sprechpraktiken und historisches Sprachwissen, die sonst nur schwer zugänglich sind. Untersucht werden in diesem Zusammenhang recht unterschiedliche Subsorten wie bspw. Brief- und Stillehren, Rede- und Rhetorikratgeber (vgl. etwa Nickisch, 1969; Schmidt-Wächter, 2004) oder Anstandslehren und Komplimentierbücher (vgl. etwa Linke, 1988, 1996). Sprachratgeber aus der jüngeren Zeit, also etwa aus dem späten 20. Jahrhundert oder sogar 21. Jahrhundert, spielen dagegen eine eher untergeordnete Rolle: Hierzu finden

${ }^{1}$ Die Überlegungen gehen aus einem größer angelegten Projekt mit kulturtheoretischem Schwerpunkt hervor, das von der VolkswagenStiftung gefördert wird. Der Name des Projektes lautet: Ein zahnloser Tiger? Über die Ent-Kritisierung des wissenschaftlichen Subjekts.

${ }^{2}$ Genau genommen müsste man wohl von Sprach- und Kommunikationsratgebern sprechen, worauf ich hier der Einfachheit halber verzichten möchte. Eine nähere Begriffsbestimmung zu Sprachratgebern erfolgt weiter unten.

${ }^{3}$ Einen sehr guten Überblick über den Forschungsstand bietet Katja Kessel (2009, S. 22 ff.). 
sich bspw. die Arbeiten von Gerd Antos (1996, 2001), Albrecht Bremerich-Vos (1991) und Katja Kessel (2009) ${ }^{4}$. In diesem Rahmen scheint der methodische Schwerpunkt - in erster Linie - darauf zu liegen, ein synchrones Bild hinsichtlich des jeweiligen interessierenden Gegenstandes zu zeichnen. Systematische diachrone Untersuchungen mit Gegenwartsbezug liegen dagegen eher nicht vor, wobei hier mit systematisch gemeint ist, dass sich das Kerninteresse der jeweiligen Fragestellung bereits entsprechend explizit in der methodischen Textauswahl niederschlagen würde ${ }^{5}$. Katja Kessel etwa nimmt in ihrer grundsätzlich synchron ausgerichteten und gegenwartsbezogenen Studie zu Kommunikationsratgebern zwar gegen Ende noch einen historischen Fokus ein, um sich auf die „Suche nach den Anfängen“ (Kessel, 2009, S. 227) bei Gesprächs- und Anstandslehren zu begeben. Dies scheint jedoch in erster Linie exemplarischen Charakter zu haben und nicht den Kern ihrer Überlegungen darzustellen ${ }^{6}$. Der untersuchte Zeitraum von der Mitte des 19. bis zum Ende des 20. Jahrhunderts wird vor diesem Hintergrund mit Blick auf die Textauswahl denn auch nur sporadisch abgedeckt und insofern auch nicht wirklich systematisch analysiert (vgl. Kessel, 2009, S. 228 f.).

Neben diesem sprachhistorischen Akzent zeigt sich aus meiner Sicht ein weiterer Schwerpunkt bei der Erforschung von Sprachratgebern: Fast naturgemäß rückt bei der Beschäftigung mit Ratgebertexten die Frage nach der Normierung bzw. der Präskription in den Vordergrund. Als eine Form der Instruktionstexte zeichnen sich Ratgeber durch die Grundfunktionen der Information und der Appellation aus (vgl. Möhn, 1991; auch Greule \& Kessel, 2009, S. 2339 f.) ${ }^{7}$. Gerade mit Blick auf die appellative Funktion wird deutlich, dass es gewissermaßen zu den Kernaufgaben von Ratgebertexten gehört, auf ihre Rezipienten einzuwirken, diese (an-) zu leiten, wie auch aus der folgenden pointierten Definition von Sprachratgebern von Albrecht Greule hervorgeht: „Ein Sprachratgeber ist ein Buch, in dem Ratschläge zum Gebrauch der Muttersprache gegeben werden oder in dem zum Gebrauch der Muttersprache angeleitet wird" (Greule, 2000, S. 318; Hervorhebung von R.N.). In diesem appellativen Anleitungscharakter von (Sprach)Ratgebertexten steckt offensichtlich eine gewisse Tendenz zur Normativität und so verwundert es nicht, dass die Frage nach der Norm sowie nach dem Ausmaß

${ }^{4}$ Mit Bezug auf Stillehren stellen etwa Greule und Kessel (2009, S. 2338) fest, dass ,,besonders Stillehren des 20. und 21. Jhs. [...] kaum beachtet" (Greule \& Kessel, 2009, S. 2338) wurden.

${ }^{5}$ Eine interessante Ausnahme stellt Law (2007) dar.

${ }^{6}$ Hiermit möchte ich natürlich nicht behaupten, dass die historische Analyse bei Kessel in irgendeiner Form undurchdacht sei. Ich möchte lediglich andeuten, dass es sich bei ihrer Arbeit nicht um eine zentral diachron angelegte Studie handelt.

${ }^{7}$ Greule \& Kessel weisen mit Bezug auf Schmidt-Wächter (2004, S. 78 ff.) darauf hin, dass man mit Blick auf Stillehren auch von einer Unterhaltungsfunktion ausgehen müsste. Sie sehen - bei Stillehren gegenwärtig sogar eine Dominanz der „,delactere“ (d. h. Unterhaltung im Sinne von Vergnügen bereiten) (Greule \& Kessel, 2009, S. 2339). Möglicherweise steht aber diese Unterhaltungsfunktion nicht neben den Grundfunktionen Information und Appellation. Vielleicht lässt sie sich vielmehr eher als Teil der appellativen Funktion betrachten, in dem Sinne, dass das Unterhalten eine Variante des Einwirkens und Leitens von Personen darstellt. 
der Normativität in Ratgebertexten stets von neuem gestellt wird, wie die folgenden zwei Beispiele von Montandon und Antos zeigen (vgl. dazu auch Kessel, 2009, S. 23):

Oft fragt man sich, ob es sich dabei um eine einfache Beschreibung von Verhaltensweisen handelt (und deren Regelung) oder ob die Bücher, weit davon entfernt, Verhaltensweisen darzustellen, ein Ideal anstreben und Regeln formulieren, die zwar als Richtpunkte dienen, aber noch längst nicht erreicht sind. (Montandon, 1991, S. 7; Hervorhebungen von R.N.)

Bei seiner Besprechung des jeweiligen Verhältnisses von Linguistik oder „Laien-Linguistik“ zu Normativität bzw. Präskription hebt Gerd Antos grundsätzlich - und wohl zurecht - hervor, dass die „Laien-Linguistik“ nicht per se und absolut präskriptiv bzw. normativ und die Linguistik demgegenüber auch nicht per se und absolut deskriptiv sei: „Präskriptivität im allgemeinen und Normativität im speziellen ist etwas, woran beide Linguistiken - wenn auch in sehr unterschiedlicher Gewichtung und Funktion - teilhaben“" (Antos, 1996, S. 22). Vor dem Hintergrund dieser differenzierten Betrachtung spricht Antos der „Laien-Linguistik“, und demnach also etwa Sprachratgebern, die Rollen als sowohl „Normüberwacher" wie auch „Normsetzer" (Antos, 1996, S. 20) zu.

Was bei der Frage nach der Normativität von Ratgebertexten insgesamt auffällt, ist nicht nur die offensichtliche Übereinstimmung darüber, dass Ratgeber - in irgendeiner Form - irgendetwas mit Normativität zu tun haben müssen. Neben diesem Einklang zeigt die Beschäftigung mit dem Thema allerdings auch eine gewisse Diffusion, was die begriffliche und terminologische Einordnung betrifft: Aus meiner Sicht macht es durchaus jeweils einen Unterschied, ob man in diesem Zusammenhang etwa von Deskription, von Darstellung, von Normativität, von Präskription, von Regelung bzw. Regelformulierung oder von Idealisierung spricht ${ }^{8}$. Diffusion besteht $\mathrm{m}$. E. auch darin, worauf die Zuschreibung der Normativität jeweils gerichtet ist: Geht es um die Normativität der Texte insgesamt? Um einzelne Textabschnitte? Um einzelne Sätze? Oder geht es etwa um so etwas wie die Intention des Autors? Damit zusammen hängt die Frage, woran man die Normativität konkret - also auf der Oberfläche - festmachen kann: An einzelnen grammatischen Formen? An Formulierungen? An Sprechakten? Oder an den in Vorworten zum Ausdruck gebrachten Absichten des Autors? Woran genau macht man also fest, dass und inwiefern ein Ratgebertext normativ ist? Es besteht in diesem Zusammenhang also offensichtlich ein gewisser Bedarf an mehr Präzisierung.

Sowohl die skizzierte Grundproblematik bei der Frage nach Normativität als auch die sprachgeschichtliche Schwerpunktsetzung stellen Ansatzpunkte für die Be-

${ }^{8}$ Dies soll jedoch keineswegs suggerieren, dass die einzelnen Ansätze (also etwa Antos) in sich immer eine inkohärente Begriffs- und Terminologieverwendung aufweisen würden. Mir geht es hier vielmehr um das Forschungsfeld zu Ratgeberliteratur insgesamt. Im Übrigen wird auch in dem vorliegenden Beitrag das Problem einer heterogenen Begriffs- und Terminologiewahl nicht gelöst. Dies würde größeren argumentativen Aufwand benötigen, der hier nicht zu leisten ist. Der obige Hinweis auf die Diffusion dient also in erster Linie dem Aufzeigen einer offenkundig recht weitgehenden Grundproblematik bei diesem Gegenstand. 
schäftigung mit Ratgeberliteratur in dem vorliegenden Beitrag dar. Hinzu kommt ein dritter Aspekt, der bei der Beschäftigung mit Ratgeberliteratur grundsätzlich zu wenig beachtet zu werden scheint: Offenbar liegt der Hauptfokus der linguistischen Untersuchungen auf Sprachratgeber und somit auf der Vermittlung von Sprachwissen an interessierte Laien. Demgegenüber scheint die Aufarbeitung von Ratgeberliteratur, die sich nicht mit dem Gegenstand Sprache und Kommunikation beschäftigt, weniger zentral zu sein, wie auch Katja Kessel betont: „Sprachratgeber sind ein Untertypus der Sachbuchgattung Ratgeber. Auch die Ratgeber allgemein sind noch nicht sprachwissenschaftlich untersucht" (Kessel, 2009, S. 24, Anm. 17). Dieses linguistische Desinteresse ist insofern ein wenig verwunderlich, als Ratgeberliteratur gegenwärtig - in einer sich zunehmend ausdifferenzierenden, spezialisierenden und als hochkomplex empfundenen Gegenwart - in den westlichen Gesellschaften mehr und mehr Relevanz zu besitzen scheint. Salopp formuliert: Ratgeber gibt es gegenwärtig für alles und jeden. Sie scheinen in diesem Zusammenhang ein gewisses Bedürfnis nach Orientierung und Leitung zu befriedigen.

Vor diesem Hintergrund sollen in dem vorliegenden Beitrag - zumindest ansatzweise - Überlegungen zu einer Studie vorgestellt werden, in der eine gegenwartsbezogene, diachrone Untersuchung von Ratgebern zum wissenschaftlichen Arbeiten vorgenommen wurde. Bei diesen Texten handelt es sich nur teilweise um Sprachratgeber im engeren Sinne, da in ihnen zwar auch mitunter das Verfassen wissenschaftlicher Texte behandelt wird. Streng genommen gehen diese Texte aber über die Vermittlung von Sprach- und Kommunikationswissen hinaus und sind insofern vielleicht eher als allgemeine, nicht-sprachliche Ratgeber im obigen Sinne zu betrachten. Der Hauptfokus soll hier auf der Frage nach der Normativität solcher Wissenschaftsratgeber liegen. Die genauen Hintergründe seien im Folgenden dargelegt.

\section{Wissenschaftsratgeber und Norm(-ierung)}

Die folgenden Ausführungen gehen auf Überlegungen zurück, die ich in Niemann (i. V.) vorgestellt habe. Ebenso wie dort schließe ich hier an der interessanten Untersuchung zu Anleitungen zum wissenschaftlichen Arbeiten von Kirsten Adamzik und Christa Pieth (1997) an, wobei ich mich im Folgenden ausschließlich auf den Normativitätsaspekt konzentrieren werde. Es geht hier also weniger darum, die Überlegungen von Adamzik und Pieth erschöpfend zu diskutieren. Vielmehr sollen ausgehend von ihren Ausführungen zu Norm und Normativität ein Mehrebenenansatz vorgestellt und speziell eine Perspektive hinsichtlich normierender Textstrukturierungsmuster skizziert werden.

Die im Folgenden zu diskutierenden Phänomene stellen einen kleinen Ausschnitt aus einer größer angelegten Studie dar, in der Wissenschaftsratgeber aus der Zeit vor 1970 mit ebensolchen aus den Jahren um 2010 systematisch und aus verschiedenen 
Perspektiven analysiert und in einem kulturtheoretischen Rahmen diskutiert werden. Die Bearbeitung des spezifischen Gegenstands der vorliegenden Untersuchung ist in diesem Rahmen noch nicht weit fortgeschritten, weshalb die folgende Darstellung eher skizzenhaften, illustrativen und weniger systematischen Charakter hat.

\subsection{Vielschichtigkeit der Normierung}

In der insgesamt sehr instruktiven (kontrastiven) Studie von Pieth und Adamzik (1997) wird unter anderem auch der Frage nach der Normativität nachgegangen: „Da Anweisungstexte Regeln formulieren, stellt die Frage, wer die normsetzende Instanz ist, wie sie eingebracht wird und mit welcher Verbindlichkeit die Anweisungen formuliert werden, einen wichtigen Faktor bei der Beurteilung dar" (Pieth \& Adamzik, 1997, S. 47 f.). Dafür richten die Autorinnen einen zentralen Blick unter anderem auf die „Normvorstellungen“ der Autoren von Wissenschaftsratgebern, indem sie die selbst-reflexiven Darstellungen in den Vorworten betrachten. Es geht dabei also um die metakommunikative Selbsteinschätzung der Autoren, inwiefern sie ihre Ratgeberausführungen als normativ bindend verstanden wissen wollen. Neben diesem selbstreflexiven Blick wird in der Studie auch analysiert, wie Aufforderungen formuliert sind, d. h., die Normativität wird an einzelnen sprachlichen (bzw. grammatischen) Formen festgemacht. Pieth und Adamzik gehen dabei davon aus, dass die „Verbindlichkeit der Normen [...] auch daran erkennbar [ist], wie die Anweisungen im einzelnen formuliert sind" (Pieth \& Adamzik, 1997, S. 49). Offensichtlich recht beliebte grammatische Mittel sind in diesem Zusammenhang etwa Imperative, Halbmodale, deontische Modalverben etc. (vgl. Pieth \& Adamzik, 1997, S. 53).

Ausgehend von diesen Überlegungen zu Normen und Normierung in Wissenschaftsratgebern habe ich in Niemann (i. V.) erörtert, inwiefern gerade isolierte Perspektiven auf einzelne Ebenen, also etwa die Ebene der Lexik oder der Morpho-Syntax, bei der Frage nach der Normativität von Wissenschaftsratgebern zu trügerischen Ergebnissen führen können. Ohne dies hier im Einzelnen nachzeichnen zu können, möchte ich zur Illustration dieser Problematik lediglich kurz auf zwei Beispiele aus meiner Studie hinweisen:

a) Mit zäher Ausdauer, die sich durch anfänglichen Mißerfolg nicht in der Überzeugung von der Macht der Wiederholung beirren läßt, kann Außerordentliches erzwungen werden. Aber das ist im Grunde Sache des Willens. Das sollte niemand über den Ankündigungen noch so besonnen vorgehender Verfasser vergessen. Auch die gediegenste, Willensschulung ${ }^{6}$ erspart doch keinem die Mühe der Selbstüberwindung. (1970er-Heyde, 3)

b) Schlagen Sie doch mal den Begriff, Prokrastination“ nach, das ist, was Sie gerade tun. Besser ist: Begrenzen Sie Ihre Tätigkeiten. Verbieten Sie sich nicht, den Fernseher einzuschalten, aber stellen Sie sich einen Wecker [...] auf eine bestimmte Zeit, dann machen Sie die Glotze wieder 
aus. Teilen Sie den Tag auf in Zeitfenster fürs Putzen und klare Zeiten für die Abschlussarbeit ${ }^{9}$. (2010er-Weber, S. 210)

Diese Gegenüberstellung soll aufzeigen, inwiefern isolierte Perspektiven auf Normativität zu einseitigen Ergebnissen führen können: Macht man etwa das Normierungspotential eines Textes morpho-syntaktisch fest an der Häufigkeit der verwendeten Imperative bzw. funktionalen Äquivalente, dann würde Beispiel $b$ als sehr normativ und Beispiel a dagegen als nicht normativ gelten. Bestimmt man demgegenüber das normierende Potential anhand der verwendeten lexikalischen Mittel, dann könnte man Beispiel a wegen der Dominanz von Wörtern mit Willens- und Zwangssemantik als normativ und Beispiel $b$ dagegen nicht normativ einordnen. Offensichtlich kann die Frage nach der Normativität bei Wissenschaftsratgebern nicht über isolierte Perspektiven beantwortet werden. Es sind folglich mehrere Ebenen zugleich zu berücksichtigen und vor allem auch weitere Ebenen, die globaler ansetzen. Was aber ist damit gemeint?

Vor dem Hintergrund der - hier nur angedeuteten - Problematik habe ich mich in Niemann (i. V.) für ein Mehrebenenmodell ausgesprochen, das man wie folgt anordnen könnte:

\begin{tabular}{|c|c|c|c|}
\hline \multicolumn{4}{|c|}{ Wie wird das Thema (Was?) präsentiert? } \\
\hline 1 & Inhalt & $\begin{array}{l}\text { - Zeitplanung vs. Zeitmanagement } \\
\text { (Thema: Umgang mit Zeit) } \\
\text { - kritische Neuschöpfung vs. } \\
\text { Eigenleistung (Thema: Originalität und } \\
\text { Kritik) }\end{array}$ & Makro \\
\hline 2 & Textstruktur & $\begin{array}{l}\text { - Persuasive Strategien in } \\
\text { Themenentfaltung } \\
\text { - bspw. Toleranz-Idealmaßstab-Kontrast vs. } \\
\text { Realisierbarkeits-Fraternisierung }\end{array}$ & \\
\hline 3 & Lexik & $\begin{array}{l}\text { - Überwindungssemantik (bei Unlust) } \\
\text { • bspw. ,Wille“,,,Stärke“"vs. ,Reflexion“, } \\
\text { "Strategie“",,Übung" }\end{array}$ & \\
\hline 4 & Morpho-Syntax & $\begin{array}{l}\text { - Deskriptiver vs. Präskriptiver Duktus } \\
\text { - bspw. deontische Modalität, Imperative, } \\
\text { usw. }\end{array}$ & Mikro \\
\hline
\end{tabular}

Abb. 1: Mehrebenenmodell zur Normativität von Wissenschaftsratgebern (vgl. Niemann i. V.)

Ohne die Hintergründe des Modells im Einzelnen zu besprechen, lässt sich als Kernidee festhalten, dass die Normierung bzw. Normativität in Wissenschaftsratgebern über ein Zusammenspiel mehrerer Ebenen bestimmt werden müsste. Diese Ebenen lassen sich differenzieren in Mikro- und Makroperspektiven: Normiert werden kann demnach auf Mikroebenen wie der Ebene der Morpho-Syntax oder aber auf globaleren

\footnotetext{
${ }^{9}$ Mit der Zitierweise 1970er-Name oder 2010er-Name soll die Einordnung der analysierten Texte in die jeweiligen Zeiträume angezeigt werden.
} 
Makroebenen wie der Ebene des Inhalts. In Niemann (i. V.) habe ich - vor dem Hintergrund einer subjektivierungstheoretischen Gesamtargumentation - dafür plädiert, dass die Frage nach der Normativität in Wissenschaftsratgebern nicht allein mit Blick auf mehrere, interagierende Ebenen gestellt werden müsste, sondern darüber hinaus auch ausgehend von globaleren Ebenen wie der Ebene des Inhalts, um auf diese Weise die oben aufgezeigte Problematik zu umgehen und außerdem nicht vorschnell Interpretationsspielraum zu verschenken. Was ist damit wiederum gemeint?

In der von mir vorgenommenen diachronen Untersuchung von Wissenschaftsratgebern hat sich herausgestellt, dass Ratgeber aus einer Zeit vor 1970 anders funktionieren als gegenwärtige. Kurz gefasst: Es zeigen sich historisch recht unterschiedliche Wege in Wissenschaftsratgebern, „Nichtwissenschaftler zu Wissenschaftlern zu machen, sie also in die Wissenschaftsgemeinschaft zu integrieren“(Pieth \& Adamzik, 1997, S. 31; Hervorhebung von R.N.). Oder anders formuliert: Es entstehen jeweils unterschiedliche wissenschaftliche Subjekte, die auf unterschiedliche Weise geformt werden. Ein Kernunterschied lässt sich dabei mit Blick auf die jeweils unterschiedlichen Grundorientierungen ausmachen: Ältere Ratgeber folgen einer Idealorientierung, jüngere einer Realorientierung. In älteren Wissenschaftsratgebern wird die wissenschaftliche Subjekthaftigkeit in der Regel in Form von Idealisierungen hergestellt, während in gegenwärtigen Ratgebern eher ein - strategisch-managerialer - Blick auf die realen und realisierbaren Umstände gerichtet wird (vgl. Niemann i. V. 2019). Um diese Grundunterscheidung in der Subjekt-Formung herauszustellen war es zunächst einmal nötig, die globale Ebene des Inhalts zu untersuchen, ohne vorab den Blick durch Analysen auf die Ebenen der Morpho-Syntax oder der Lexik möglicherweise zu verstellen.

Im vorliegenden Beitrag möchte ich daran nun mit einer weiteren Perspektive anschließen: Wie aus dem obigen Modell hervorgeht, werden für die Bestimmung der Normativität von Wissenschaftsratgebern nicht nur - wie üblicherweise - grammatische Kategorien oder lexikalische Aspekte berücksichtigt. Neben diesen Ebenen und der Ebene des Inhalts scheint in diesem Zusammenhang auch die Betrachtung von bestimmten Textstrukturen lohnenswert zu sein: Noch präziser formuliert macht es den Eindruck, als schlüge sich die - auf der Inhaltsebene ermittelte - Subjektivierungsunterscheidung ideal vs. real auch in der Art und Weise der Gestaltung mancher Textabschnitte nieder, und zwar insofern, als sich bestimmte Muster der Themenentfaltung zeigen, in denen die Grundunterscheidung ideal vs. real eine zentrale Rolle spielt und die insofern gewissermaßen als Strategien der Subjektivierung interpretiert werden können. Dies sei im folgenden Abschnitt skizziert.

\subsection{Normierende Strategien in der Textstruktur}

Im Folgenden seien also solche Textabschnitte einmal näher betrachtet und vor allem mit Blick auf die Frage nach dem strategischen Gehalt diskutiert. Es sei noch einmal betont, dass es sich bei den folgenden Ausführungen lediglich um erörternde, 
noch in Arbeit befindliche Überlegungen handelt. Es geht mir im Folgenden - wie bereits betont - also eher um eine skizzenhafte Erörterung.

Die beiden gewählten Beispiele stammen zum einen aus einem älteren und zum anderen aus einem jüngeren Wissenschaftsratgeber. Bei beiden geht es thematisch um die Zielsetzung, die mit dem Erwerb von Kompetenzen beim wissenschaftlichen Arbeiten verbunden ist oder sein könnte. Beginnen wir mit dem älteren Textauszug:

c) Darüber müssen Sie sich einmal in einer stillen Stunde ganz klarwerden. Kaum etwas könnte den Erfolg Ihrer Arbeit mehr beeinträchtigen als eine Selbsttäuschung über Ihre Absichten. Eine Arbeit kann nur erfolgreich sein, wenn sie ein klares Ziel hat. Dieses Ziel wird aber nicht schon mit dem Thema bestimmt, dazu gehören auch die Beweggründe Ihrer Arbeit. Ich weiß, daß diese Frage nicht immer leicht zu beantworten ist, denn Selbsterkenntnis ist weder einfach noch beliebt. Aber es hilft nichts, Sie müssen sich hindurchringen, es erspart Ihnen später wirklich viele Schwierigkeiten, Umwege und Enttäuschungen.

Es gibt so viele Gründe: Geldverdienen, Einfluß und Ansehen gewinnen, eine bessere Stellung erreichen, selbstlos einer Sache dienen. Keiner dieser Gründe ist an sich schon besonders gut oder schlecht. Leichthin wird manchmal der Schriftsteller, der offen zugibt, daß er vom Ertrag seiner Arbeit leben will und muß, etwas verachtet. Das ist falsch, denn entscheidend ist doch, ob seine Arbeit ernsthaft zu werten ist. Wer oberflächlich arbeitet, heute über Blumenzucht und morgen über Julius Cäsar, gehört natürlich nicht zum Kreis der ernsthaft zu wertenden Geistesarbeiter. (1970er-Kliemann, S. 127)

In diesem Textabschnitt scheint mir eine eigentümliche Zweiteilung ${ }^{10}$ vorzuliegen: Der Autor beginnt seine Ausführungen mit dem Hinweis, dass man sich über seine Ziele beim wissenschaftlichen Arbeiten bewusst werden müsse. Er hebt den Stellenwert dieser Bewusstmachung hervor, indem er betont, dass klare Zielsetzungen über den ,Erfolg“6 der Arbeit entscheiden. Anschließend stellt er dem Leser dies noch mit einer weitergehenden Begründung dar, die mit einer Konzession gegenüber einer vermeintlichen Lesereinstellung beginnt, die vom Autor antizipiert wird („Ich weiß, daß... Aber...“). Mit dieser konzessiven Antizipation der Lesereinstellung zeigt der Autor Bereitschaft, die realen Voraussetzungen anzuerkennen und darauf einzugehen. Auf diese Weise erzeugt er eine gewisse Nähe zum Leser. Daraufhin folgt in einem neuen Absatz eine Auflistung verschiedener potentieller „Gründe“ für wissenschaftliches Arbeiten, mit dem Zusatz, dass im Grunde alle genannten „Gründe“ ihre Berechtigung besitzen und keiner ,,besonders gut oder schlecht“" sei. Der Autor unterstreicht hier also offenkundig ein hohes Maß an Toleranz, das mit einem betont realistischen (und wertfreien) Blick auf die sehr vielseitige Motivlage zum wissenschaftlichen Arbeiten einhergeht.

Diese realistische und tolerante Haltung scheint ganz plötzlich ins Gegenteil umzuschlagen, wenn der Autor mit „Das ist falsch, denn“ die aus seiner Sicht wichtigste Voraussetzung beim wissenschaftlichen Arbeiten einleitet: Mit der Betonung der Ernsthaftigkeit nimmt der Autor nun explizit Stellung. Er macht - unmissverständ-

${ }^{10}$ Diese textuelle Zweiteilung ist durch die graue Markierung angezeigt. 
lich - klar, was als Ideal eines wahrhaftigen „Geistesarbeiters“ gilt. Diese wertsetzende Positionierung wird besonders gut daran ersichtlich, dass der Autor hier mit ernsthaft vs. oberflächlich eine Dichotomisierung vornimmt, mit der ein eindeutiges Definitionskriterium für einen imaginierten (und als offensichtlich erstrebenswerten) „Kreis von Geistesarbeitern“ bestimmt wird. Im Vergleich zu den vorausgehenden Ausführungen entsteht durch diese Darstellung nun ein relativ starker Bruch, der sich als Toleranz-Idealmaßstab-Kontrastierung bezeichnen lässt. Diese Kontrastierung lässt sich so interpretieren, dass gerade durch die anfängliche realistisch-tolerante Haltung, mit der der Autor Authentizität und eine gewisse Nähe zum Leser etabliert, die Idealsetzung im letzten Teil besonders markant hervorsticht. Die Idealorientierung, die ja - wie gesagt - für die älteren Wissenschaftsratgeber insgesamt charakteristisch zu sein scheint, tritt auf diese Weise umso deutlicher hervor, sie wird so in ein besonders grelles Licht gerückt.

Derartige Kontrastierungen scheint es in den älteren Wissenschaftsratgebern häufiger zu geben. Der Autor gibt sich dabei stets zunächst tolerant und deutet damit dem Leser an, dass er die realen Umstände anerkennen und nicht vorschreibend agieren möchte. Im Anschluss an diese anfängliche Unbestimmtheit geht er dann über zu eindeutigen (etwa moralischen) Wertsetzungen, um auf diese Weise ein Ideal von wissenschaftlicher Subjekthaftigkeit zu etablieren. Diese Idealisierungen wiederum erscheinen normativ. Das folgende, nicht näher zu besprechende Beispiel soll der Illustrierung dessen dienen:

d) Vorschriften im einzelnen geben zu wollen verbietet sich, wenigstens für alle Fragen der Lebensweise, bei der persönlichen Verschiedenheit der Menschen ohnehin von selbst. Jeder muß eine vernünftige, ihm zuträgliche Lebensform selbst erproben. Allerdings - Körperstählung ist für jedermann ebenso unerläßlich wie die Befolgung des Ratschlags Goethes: ,Der Geist muß nur nicht dem Körper nachgeben‘. (1970er-Heyde, 2)

Hinter dem aufgezeigten Muster lässt sich aus meiner Sicht ein strategisches, rhetorisches Vorgehen erkennen, das im Kern auf die Autor-Leser-Beziehung zielt: Der Autor erzeugt durch sein tolerant-realistisches Auftreten zunächst Authentizität und Glaubwürdigkeit gegenüber dem Leser. Es wird eine Atmosphäre des Vertrauens aufgebaut, in der die anschließende normierende Idealisierung besonders wirksam ist.

Wie verhalten sich demgegenüber die gegenwärtigen Wissenschaftsratgeber? Auch bei ihnen lassen sich textuelle Muster ausmachen, die als Strategien im obigen Sinne interpretiert werden können. Ihre Stoßrichtung scheint dabei allerdings eine etwas andere zu sein und stärker mit der generellen gegenwärtigen Realorientierung einher zu gehen.

e) Je deutlicher Sie vor Augen haben, was Sie selbst von sich und Ihrer Arbeit erwarten und was Sie bereit sind, für ein gutes Ergebnis zu tun, desto genauer lassen sich die Arbeitsschritte planen und der Zeitaufwand abschätzen.

Natürlich schreit nun eine ganze Riege von Professoren auf, die annehmen, jeder Diplomand sei Akademiker, jeder Akademiker Wissenschaftler und jeder Wissenschaftler stets Bestleistungen 
verpflichtet. In der Realität aber sollen Sie mit 25 fertig sein, 2 Jahre im Ausland verbracht haben, Praktika in Zeugnisform stapelweise vorweisen, biegbar und flexibel, aber auch gestanden und erfahren sein. Und vor allem wissen, wovon Sie reden.

Die Abschlussnote [...] kann bei all den Nebenbedingungen unter Umständen in den Hintergrund treten. So mancher spätere Chef schaut aber dennoch auf Thema und Note, hier liegen Chancen, die Sie nicht vergeben sollten. Wichtig ist in jedem Fall, dass Sie verstanden haben, was Sie da schreiben, dies sollte der Minimalanspruch an eine Studienabschlussarbeit sein, weitere können sich ergeben. (2010er-Weber, 37)

Auch in diesem Beispiel geht es thematisch darum, sich über die eigenen Ziele beim wissenschaftlichen Arbeiten klar zu werden. Die Autorin gibt sich dabei von Beginn an sehr realistisch: Sie betont, dass die Ratsuchenden sich ,vor Augen' führen sollten, was sie , selbst von sich ' und ihrem wissenschaftlichen Arbeiten, erwarten'. Es gehe also darum, in sich hinein zu hören und die eigenen, wirklichen Bedürfnisse zu überprüfen, um zu bestimmen, wie viel man leisten bzw. investieren möchte. Es handelt sich hier im Grunde um eine ganz realistische Kosten-Nutzen-Kalkulation: Das Maß dessen, was man investiert, richtet sich nach ganz individuellen Zielen und Ansprüchen. Dieser kalkulatorische Charakter steht einer - bis dato nur angedeuteten - idealisierten Vorstellung gegenüber, bei der pauschal angenommen und vorausgesetzt wird, dass man die beste und vollkommene wissenschaftliche Leistung erbringen möchte.

Dieser sehr realistische Fokus auf das wissenschaftliche Arbeiten wird daraufhin im nächsten Abschnitt verschärft: Die Autorin imaginiert dabei zunächst einmal eine ,ganze Riege von Professoren', die bei so viel Realitätssinn angeblich ,aufschreien“ würde, weil doch beim wissenschaftlichen Arbeiten ,stets Bestleistungen ' anzustreben seien. Dieser idealisierten, beschönigten Sichtweise der ,Professoren` setzt die Autorin daraufhin die Realität der studentischen Welt entgegen, indem sie die realen Anforderungen und Erwartungen formuliert, denen Studierende sich heutzutage ausgesetzt sehen. Die Autorin inszeniert sich hier als eine Instanz, die eine - angeblich - normalerweise verschwiegene Realität deutlich und frei heraus benennt, ohne diese zu beschönigen oder zu verklären. Sie positioniert sich auf diese Weise gegen einen vermeintlichen professoralen Mainstream und solidarisiert bzw. fraternisiert sich gleichzeitig mit ihren Lesern.

Im darauffolgenden Abschnitt zieht die Autorin dann zunächst einmal den Schluss aus den vorausgehenden Ausführungen, indem sie konstatiert, dass all diese realistischen Umstände , unter Umständen' dazu führen, dass die Abschlussnote bei den Studierenden nicht im Vordergrund steht. Ein wenig widersprüchlich dazu fährt sie mit dem - ebenfalls wieder sehr realistischen - Hinweis fort, dass „so mancher spätere“ Chef aber vielleicht doch auf die Abschlussnote schauen würde und dass dies eine Chance sei, die man nicht ,vergeben sollte“. Sie schließt mit der Betonung eines „Minimalanspruchs“, den man beim wissenschaftlichen Arbeiten haben sollte. Er besagt recht vage, dass man ,verstanden haben“"sollte, was man da schreibt. Auch mit dieser abschließenden Zielformulierung wird eine außerordentliche Realitäts- und Realisierbarkeitsorientierung zum Ausdruck gebracht. 
In diesem Beispiel liegt ebenfalls - wie oben - so etwas wie eine Beziehungspflege seitens der Autorinstanz vor: Die Autorin verschafft sich mithilfe ihres entlarvenden Gestus einerseits auch hier eine gewisse Authentizität. Aber darüber hinaus erzeugt sie durch die Aufdeckung des angeblichen Mainstreamdenkens andererseits noch eine Art Gegen-Gemeinschaft, die sich durch ein klares Wir-Die-Schema definiert: In dieser Dichotomie stehen auf der einen Seite die realitätsfremden Professoren und Wissenschaftler und auf der anderen Seite die realistischen Studierenden (und natürlich die Autorin als deren Sprecherin).

Mit der Fraternisierung macht sich die Autorin also zu einem Bestandteil ihrer Lesergemeinschaft; diese Rolle nimmt sie ein, indem sie auf die studentische Realität und die Realisierbarkeit pocht. Vor diesem Hintergrund könnte man hier auch von einer Realisierbarkeits-Fraternisierung sprechen: Dieses Muster funktioniert in dem Sinne, dass ein Autor eine Autor-Leser-Gemeinschaft etabliert, die darauf gründet, dass er seiner Leserschaft eine Art realistischen Spiegel vorhält. Dazu gehört zugleich die Abgrenzung von einem Außen, das nicht zu dieser Autor-Leser-Gemeinschaft gehört. Dieses Außen wird hier negativ als realitätsferne, idealistische Träumerei und insofern als zu hohe, nicht-realisierbare Erwartung präsentiert. Die Idealisierung wird hier demnach als Ausgangspunkt zur Dichotomiebildung und insofern zur Etablierung der Gemeinschaft genutzt. Auch dieser Fall des Realisierbarkeits-Fraternisierungsmusters lässt sich aus meiner Sicht als eine normierende Strategie interpretieren, die in jüngeren Wissenschaftsratgebern häufiger anzutreffen ist.

\section{Fazit}

Die besprochenen Textstellen lassen sich in der Form interpretieren, dass hier offensichtlich historisch unterschiedliche textuelle Strategien der Subjektivierung vorliegen; sie bringen in diesem Sinne eine gewisse Normierung zum Ausdruck, die sich je nach Zeitraum unterschiedlich akzentuiert: Auf beiden Seiten fügen sich die Beispiele zunächst einmal jeweils in die typischen Subjektivierungsweisen ihrer Zeit ein, die auf der Ebene des Inhalts mit den Schwerpunkten einer Ideal- vs. Realorientierung festgemacht werden konnten. Das Muster der Toleranz-Idealmaßstab-Kontrastierung betont die für die älteren Ratgeber übliche Idealisierung, während die Realisierbarkeits-Fraternisierung die Realorientierung der jüngeren Wissenschaftsratgeber bedient.

Interessant ist in diesem Zusammenhang, dass offensichtlich bei beiden Mustern mit Dichotomien gearbeitet wird, die sich in textuellen Kontrastierungen niederschlagen und die für eine Art Beziehungspflege zwischen Autor- und Leserinstanz gebraucht werden. Ungeachtet der jeweiligen schwerpunktmäßigen Orientierung werden bei beiden Mustern die zwei Seiten der Ideal-Real-Dichotomie gegeneinander ausgespielt, natürlich auf jeweils spezifische Weise. Bei der Toleranz-Idealmaßstab-Kontrastierung dient der Fokus auf realistische Zustände etwa dem Ausdruck einer toleranten 
Haltung des Autors, durch die die folgende idealisierend-normierende Wertsetzung umso stärker wirkt, weil zuvor eine Atmosphäre des Vertrauens geschaffen wurde. Bei der Realisierbarkeits-Fraternisierung wiederum wird ein realitätsfernes und insofern idealisierendes Außen suggeriert, vor dessen Hintergrund sich der Autor mit seiner Leserschaft fraternisieren kann, indem er dem überzogenen, realitätsfernen Außen eine realistische und realisierbare Perspektive entgegenhält.

Mit der Beispielbesprechung sollte im vorliegenden Beitrag insgesamt angedeutet werden, inwiefern die Frage nach der Normativität bzw. der Normierung in Wissenschaftsratgebern auf unterschiedlichen Ebenen gestellt werden kann, wobei hier speziell die Ebene der textstrukturellen Musterhaftigkeit im Vordergrund stand. Es wurden dabei - in diachroner Perspektive - Muster herausgearbeitet, die sich als (persuasive) Strategien im Zusammenhang von Norm und Normierung deuten lassen. Zentral behandelt wurde im vorliegenden Beitrag vor diesem Hintergrund die Strategie der Dichotomisierung, was jedoch nicht bedeuten soll, dass sich normierende Subjektivierungsstrategien darin erschöpften. Es bleibt also zukünftigen Untersuchungen vorbehalten, diesbezüglich weitergehende und vor allem systematischere Akzente zu setzen.

\section{Literatur}

\section{Primärliteratur}

Heyde, J.E. (1933). Technik des wissenschaftlichen Arbeitens. Eine Anleitung, besonders für Studierende. 4., verb. und verm. Aufl. Berlin: Junker und Dünnhaupt.

Kliemann, H. (1965). Anleitungen zum wissenschaftlichen Arbeiten. Praktische Ratschläge und erprobte Hilfsmittel. 5., verb. Aufl. Freiburg: Rombach \& Co.

Weber, D. (2014). Die erfolgreiche Abschlussarbeit für Dummies. 2., aktual. Aufl. Weinheim: Wiley-VCH.

\section{Sekundärliteratur}

Antos, G. (1996). Laien-Linguistik. Studien zu Sprach- und Kommunikationsproblemen im Alltag. Am Beispiel von Sprachratgebern und Kommunikationstrainings. Tübingen: Niemeyer.

Antos, G. (2001). Gesprächsanalyse und Ratgeberliteratur. In K. Brinker \& G. Antos \& W. Heinemann \& S.F. Sager (Hrsg.), Text- und Gesprächslinguistik. Ein internationales Handbuch zeitgenössischer Forschung. 2. Halbband (S. 1716-1725). Berlin-New York: de Gruyter.

Bremerich-Vos, A. (1991). Populäre rhetorische Ratgeber. Historisch-systematische Untersuchungen. Tübingen: Niemeyer.

Greule, A. (2000). „Deutsch fürs Leben“. Fünfhundert Jahre Sprachratgeber. In K.M. Eichhoff-Cyrus \& R. Hoberg (Hrsg.), Die deutsche Sprache zur Jahrtausendwende. Sprachkultur oder Sprachverfall? (S. 317-329). Mannheim-Wiesbaden: Duden.

Greule, A. \& Kessel, K. (2009). Praxisbezogene Stillehren. In U. Fix \& A. Gardt \& H.E. Wiegand (Hrsg.), Handbücher zur Sprach- und Kommunikationswissenschaft. Bd. 31.2, Rhetorik und Stilistik (S. 23342349). Berlin-New York: de Gruyter.

Kessel, K. (2009). Die Kunst des Smalltalks. Sprachwissenschaftliche Untersuchungen zu Kommunikationsratgebern. Tübingen: Narr.

Law, C. (2007). Sprachratgeber und Stillehren in Deutschland (1923-1967). Ein Vergleich der Sprach- und Stilauffassung in vier politischen Systemen. Berlin-New York: de Gruyter. 
Linke, A. (1988). Die Kunst der „guten Unterhaltung“. Bürgertum und Gesprächskultur im 19. Jahrhundert. Zeitschrift für germanistische Linguistik, 16, 123-144.

Linke, A. (1996). Sprachkultur und Bürgertum. Zur Mentalitätsgeschichte des 19. Jahrhunderts. Stuttgart-Weimar: Metzler.

Möhn, D. (1991). Instruktionstexte. Ein Problemfall der Textidentifikation. In K. Brinker (Hrsg.), Aspekte der Textlinguistik (S. 183-212). Hildesheim: Olms.

Montandon, A. (Hrsg.). (1991). Über die deutsche Höflichkeit. Entwicklung der Kommunikationsvorstellungen in den Schriften über Umgangsformen in den deutschsprachigen Ländern. Frankfurt am Main: Peter Lang.

Nickisch, R.M.G. (1969). Die Stilprinzipien in den deutschen Briefstellern des 17. und 18. Jahrhunderts. Mit einer Bibliographie zur Briefschreiblehre 1474-1800. Göttingen: Vandenhoeck \& Ruprecht.

Niemann, R. i. V. Das , unternehmerische Wissenschaftler-Subjekt'? Widerspruch und Subjektivierung in der Wissenschaft betrachtet anhand von Ratgeberliteratur. Erscheint in I.H. Warnke \& S. Schattenberg \& A.-K. Hornidge \& M. Nonhoff (Hrsg.), Wiesbaden: Springer VS.

Niemann, R. i. V. (erscheint vorauss. 2019). Zum Wandel des wissenschaftlichen Subjekts. Von kritischer Wissensschöpfung zum postkritischen Selbstmangement? Bielefeld: Transcript.

Pieth, Ch. \& Adamzik, K. (1997). Anleitungen zum Schreiben universitärer Texte in kontrastiver Perspektive. In K. Adamzik \& G. Antos \& E.-M. Jakobs (Hrsg.), Domänen- und kulturspezifisches Schreiben (S. 31-69). Frankfurt am Main: Peter Lang.

Schmidt-Wächter, A. (2004). Die Reflexion kommunikativer Welt in Rede- und Stillehrbüchern zwischen Christian Weise und Johann Christoph Adelung. Frankfurt am Main: Peter Lang. 